\title{
The underlying mechanism of partial anterior cruciate ligament injuries to the meniscus degeneration of knee joint in rabbit models
}

\section{Dalin Wang}

Beihua University

\section{Zhe Wang}

Beihua University

Mingcheng Li ( $\square$ limingcheng@beihua.edu.cn )

Beihua University https://orcid.org/0000-0003-2640-4305

\section{Songbao Xu}

Research Institute of Petroleum Exploration and Development

\section{Research article}

Keywords: Anterior cruciate ligament; anteromedial bundle; posterolateral bundle; meniscus degeneration

Posted Date: June 2nd, 2020

DOl: https://doi.org/10.21203/rs.3.rs-18786/v2

License: (c) (1) This work is licensed under a Creative Commons Attribution 4.0 International License. Read Full License

Version of Record: A version of this preprint was published on September 18th, 2020. See the published version at https://doi.org/10.1186/s13018-020-01954-6. 


\section{Abstract}

Background: The diagnosis, treatment and efficacy evaluation of anterior cruciate ligament (ACL) partial rupture remains controversial. This research aims to investigate the underlying mechanism of partial ACL injuries to the meniscus degeneration in the rabbit knee.

Methods: Sixty New Zealand, white rabbits were randomly divided into three groups including an experimental group, a sham group $(n=6)$ and and a blank control group $(n=6)$. The experimental group is composed of an anteromedial bundle (AMB) rupture group $(n=24)$, a posterolateral bundle (PLB) rupture group $(n=24)$. Rabbits in the experimental group were subjected to right hind limbs knee surgery to induce ACL part injury under the arthroscopy. Finally, eight rabbits including 6 in the model group and 2 in the control group were sampled randomly on the second, fourth and eighth weeks respectively. We observed the typical form of the meniscus through HE staining. Expressions of inflammatory factors including interleukin-1 $\beta$ (IL-1 $\beta$ ) and IL-17 in the knee joint fluid were determined by means of an ELISA. Analysis of the mRNA expressions of matrix metalloproteinases-13(MMP-13) was performed to evaluate the inflammatory mediators in the pathogenesis of the meniscus.

Results: HE staining results showed that the surface was rough and the tissues were loose displaying collagen fibers of varying thickness. Both IL-1 $\beta$ and IL-17 in the synovial fluid, and the positive rate of MMP-13 in addition to MMP-13 mRNA showed a demonstrable increase treads from the 2 nd to the 8 th week. The significant difference was found $(P<0.05)$ compared to the control group.

Conclusion: We conclude that the elevated levels of IL-1 $\beta$ and IL-17, along with increased MMP13 expression, resulted in meniscus degradation in the rabbit knee joint model with partial ACL injury.

\section{Background}

The anterior cruciate ligament (ACL) is prone to be most ruptured ligament in the knee sporting injuries. With the increasing numbers of an aging population sport and the increase in traffic accidents, ACL rupture has become an extremely common injury, which accounts for about half of all knee ligament injuries in China [1]. Clinically, the severity of ACL injury is divided into three types: mild, moderate and severe rupture. In the cases of moderate and severe injuries, ACL reconstruction surgery is recommended. Injury to one-quarter of a single bundle of the $A C L$ are commonly referred to as a mild injury or termed partial ACL injury [2]. ACL partial tears, which were damaged to one of the two ACL fiber bundles, were difficult to diagnose without showing accurate joint biomechanics with MRI examination, moreover, injury assessment of its functional retention with direct arthroscopic examination also was difficult owning to its diverse physical characteristics[3,4].

The diagnosis, treatment and efficacy evaluation of the partial ACL injury remain challenging with conflicting reports in the literature [5]. When the ACL part tear develops, poor knee stability results in a greatly increased probability of joint injury. Therefore, treatment of partial ACL is related to aspects of the 
patient's knee joint, such as a meniscus, articular cartilage and other structures of acute or long-term damage [6].

Lohmander et al first evaluated the effects of different levels of cytokines in human knee joint fluid after injury to the cruciate ligament or meniscus in 1994 [7]. For the past two decades, numerous studies have reported elevated levels of inflammatory cytokines within the joint environment were associated with knee trauma in addition to knee osteoarthritis (OA) of progression [8]. Additionally, matrix metalloproteinases $\left(\mathrm{MMP}_{\mathrm{S}}\right)$ are a class of enzymes widely present in the connective tissue of the articular cartilage extracellular matrix (ECM). Physiological and pathological degradation of ECM plays an important role in the protease super-family, which can be divided into various sub-types according to dissimilar substrates. MMP-13 was selected as a bio-marker of ligament damage as it belongs to the collagenase sub-type of MMPs, which can directly degrade type 2 collagen in the cartilage matrix and reflect the metabolic changes in cartilage matrix [9].

This study was designed to establish a model of discrete partial ACL bundle injuries in the knee joint of rabbits, and to observe whether the differences in the morphology and histology of the meniscus are impacted by partial ACL different functional bundle injuries on the knee meniscus. In addition, we aimed to determine the significance of inflammatory factors in the articular fluid of meniscus degeneration. We also presented a predictive factor of partial ACL injury evolution into meniscus degeneration based on the pre-clinical examination in animal models.

\section{Methods}

\section{Animals and Groups}

This experiment was approved by the Animal Experiments Ethics Committee of the Associated Hospital of Beihua University (Ethical approval number: Protocol Number 2017-08-16). Animal care was in accordance with the Animal Research: Reporting in Vivo Experiments guidelines. The study was conducted on 60 skeletally mature New Zealand rabbits (23-weeks-old) weighing between $2.5-3.0 \mathrm{~kg}$. All healthy SPF-grade male animals were obtained from the Experimental Animal Breeding and Research Centre, Bethune Medical College Animal Experimental Center, Jilin University (No.: SCXK-2018-0006). Based on previous experience, the animals were randomly assigned into three groups using a random contrast method and random number tables including a experimental group, sham group $(n=6)$ and and a blank control group $(n=6)$. The experimental group is composed of an anteromedial bundle (AMB) rupture group $(n=24)$, a posterolateral bundle $(P L B)$ rupture group $(n=24)$.

\section{Plan-operative procedure}

Rabbits in the model group were subjected to right hind limbs knee surgery to induce ACL part injury under the arthroscopy (Andover, MA, USA; 72200616) as previously described [2, 10]. In a brief, the rabbits were fixed in a supine position on the operation table with the $4 \mathrm{~cm} \times 4 \mathrm{~cm}$ surgical area shaved. Anesthesia was administered intramuscularly at dosages of ketamine $(100 \mathrm{mg} / \mathrm{kg})$, disinfected with 
complexed iodine, draped a sterile surgical towel. An adequate opening was built with $0.5-\mathrm{cm}$ long incisions through the anterior medial and lateral approach into the joint cavity. The operator explored the intact structures of articular cartilage and meniscus. While completely bleeding was stopped and fixed the knee at $0^{\circ}$, the $A C L$ was fully exposed. In AMB rupture group, a special blunt hook was used to hold the tension fibers in the middle and lower in $1 / 2$ of the ACL, and then cut off the bundle. In PLB rupture group, firstly, flex the knee to 90 , application of the special hook is to hold the loose fiber bundle in the middle and lower $1 / 2$ of $A C L$, afterwards, cut off the fiber bundle while the knee joint is straightened. In the sham operation group, the same procedure as the medial incision of the knee into the joint cavity was exposed without cutting off the ACL. The Anterior drawer test confirmed that the model of ACL rupture had been successfully created while the incision was then sutured with $3 / 0$ absorbable sutures. Four days after surgery, all postoperative rabbits were intramuscularly injected with 400,000 U penicillin to prevent infection. In a blank group, the rabbit legs did not make any interventions with normal feeding. All rabbits were housed with sub-cage feeding. The cage size was $60 \mathrm{~cm} \times 60 \mathrm{~cm} \times 40 \mathrm{~cm}$ with a temperature of $23-25^{\circ} \mathrm{Cand}$ a relative humidity of $55 \%$.

\section{Gross observations of animal statuses and meniscus}

Animal body weight was measured, and wound conditions as well as gait were observed and recorded daily. For joint function scores, the knees behavioral scores of the three groups of animals were scored according to the absence of passive movement of the knee, the degree of lameness and the stability of the anterior drawer test, with a minimum score of 0 and a maximum score of 9 at $2^{\text {nd }}, 4^{\text {th }}$ and $8^{\text {th }}$ weeks after surgery with a reference to Sun et al [11]. Six rabbits were randomly sacrificed in the experimental group at the $2^{\text {nd }}, 4^{\text {th }}$ and $8^{\text {th }}$ weeks after the operation. And, 2 rabbits in the controls were performed as the experimental group. The knee was experimentally dissected and the typical meniscus shapes were observed along with other properties including fractures, color, and surface smoothness.

\section{Histopathological and immunohistochemistry evaluation}

The posterior angles of the medial meniscus in the three groups were cut separately and the surrounding tissue was thoroughly removed. $0.9 \%$ sodium chloride brine was rinsed and fixed for 24 hours. The histological section of the operational side of the meniscus was stained with haematoxylin-eosin. Histological changes in the meniscus tissue were measured according to the method as previously described [10].

Immunohistochemical staining using antibodies directly against MMP-13 was adopted with the Histostain-SP kit (Zymed, San Francisco, CA). The tissue sections were incubated with a specific antibody against MMP-13 (Santa Cruz Biotechnology, Inc., Santa Cruz, CA) (1:500). Normal goat IgG (1:300) was used as a negative control. The expression of MMP-13 was detected by a biotin-streptavidin-peroxidase system using diaminobenzidine as a chromogen. Counterstaining was carried out with hematoxylin.

\section{Cytological measurement of knee joint fluid samples}


Primary outcomes included levels of IL-1 $\beta$ and IL-17 in the knee joint fluid. For each operative animal, a minimum of $1 \mathrm{ml}$ of synovial fluid was aspirated from the knee joint prior to orthopedic surgery via direct needle aspiration. Samples were placed in a BD Vacutainer test tube and centrifuged at 3,000 rpm for 10 minutes. The supernatant was stored at $20^{\circ} \mathrm{C}$ until the assay. The levels of IL- $1 \beta$ and IL-17 were measured by means of an enzyme-linked immunosorbant assay (ELISA) with the Quantikine ${ }^{\circledR}$ HS Human IL-1 $\beta$ and IL-17 Immunoassay kit (R\&D Systems, MN, USA). The limit of detection was lower than $2 \mathrm{pg} / \mathrm{mL}$ for IL-1 $\beta, \mathrm{IL}-17$, and the intra-batch CV and inter-batch CV were $<9 \%$ and $<15 \%$ respectively.

\section{RNA isolation and analysis of the mRNA expressions of MMP-13}

$0.1 \mathrm{~g}$ of fresh meniscus tissue was cut into a foam with scissors. The pellets were collected and total RNA was extracted with TRIzol reagent (Invitrogen, Carlsbad, CA, USA). cDNA was synthesized with a cDNA synthesis kit (Bio-Rad, Hercules, CA, USA) according to the manufacturer's instructions. The primer sequences are as follows: MMP-13 forward TGACCACTCCAAGGACCCAG; reverse GAGGATGCAGACGCCCAGAAGA. G3PDH forward CCACTTTGTGAAGCTCATTTCCT; reverse TCGTCCTCCTCTGGTGCTCT.

qRT-PCR analysis of the mRNA was performed using a standard kit (Invitrogen, Carlsbad, CA, USA). The relative transcript levels of the target genes were normalized to that of G3PDH using the $2^{-\Delta \Delta C t}$ assay. Relative levels of gene expression are presented as the mean \pm standard deviation of three independent experiments.

\section{Statistical analysis}

Statistical analysis was performed with SPSS version 10.0 (SPSS, Inc., Chicago, IL, USA) and data are expressed as the mean \pm standard deviation. Normality of data distribution was assessed by the JarqueBera test. When the cytokine concentrations were non-Gaussian distribution, both $t$-test and MannWhitney-Wilcoxon (MWW) tests were utilized. Analysis of variance (one-way ANOVA) and post hoc Bonferroni multiple comparisons test was utilized to compare differences between groups. A value of $P<0.05$ was considered statistically significant.

\section{Results}

\section{Characteristics of animal statues and meniscus in the ALB and PLB groups}

At two weeks post-surgery, the incisions in all groups had recovered without any signs of infection or delayed healing and the control group remained healthy. Operated limbs could be gradually moved and no abnormalities were found. There was no significant difference in the functional score of the operated knee joint of rabbits in the control group at 2 weeks $(7.42 \pm 0.24 \rrbracket, 4$ weeks $(7.53 \pm 0.25 \rrbracket$ and 8 weeks (7.44 \pm 0.25 \after surgery $(P>0.05)$. In the ALB and PLB group, functional score of the operated knee

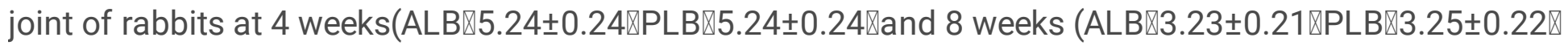
was lower than that of rabbits at 2 weeks (ALB囚6.42 $\pm 0.23 \bigotimes P L B \bigotimes 6.32 \pm 0.24 \rrbracket$ after operation $(P<0.05)$, 
and functional score of the operated knee at 8 weeks was lower than those of 4 weeks $(P<0.05)$. Compare with the same time point, functional score of the operated knee in the both model group were significantly lower than those in the control group $(P<0.05)$. Besides, there was no significant difference in knee joint function score between the two groups at time points $(P>0.05)$.

In the control groups, the structure of the meniscus was complete and the surface was bright white, very smooth, showed no tears each week. In AMB group after 2 weeks, the meniscus was smooth, dark yellow in color and had complete general structure, showed good toughness with no obvious tears on the body and the free margin. In week 4 , the general meniscus structure was still complete. The surface was rough and pale yellow with relaxation and no tears. In week 8 , the free margin on the meniscus appeared to be worn out, had a rough surface, was dark yellow, had poor toughness and the degree of tension significantly decreased. In PLB group, the meniscus had an intact structure and poor surface flatness, was pale yellow, had no tears at the second week. At the fourth week, the inner edge of the meniscus showed tear, the surface was rough, yellowish, with obvious signs of relaxation. The meniscus body was damaged, the surface flatness had significantly deteriorated, it was deep yellow, the toughness had noticeably deteriorated, and the degree of tension was significantly reduced after eight weeks.

\section{Pathological morphology of meniscus on the ALB and PLB groups}

In the control groups, the meniscus HE staining characteristics showed that the surface was dense and smooth. Chondrocyte cells and collagen fibers were arranged neatly and were well defined. Non-clustered chondrocytes, and non-angiogenesis in addition to inflammatory cells were found (Fig. 1 a). In the AMB groups, staining showed that the meniscus surface structure was consistent and complete. The collagen fibers were arranged in a compact area, and only the chondrocytes cells were enlarged (Fig. 1 b) for the 2nd week. The staining depth changed and the surface was uneven. Also, the tissue was loose and the chondrocyte cells were irregularly arranged (Fig. $1 \mathrm{c}$ ) at the 4th week. At the 8th week, the meniscus smoothness was significantly reduced, showing that the local slag formation and collagen fiber tissue sequence had altered dramatically and a small amount of inflammatory cells infiltration was observed (Fig. 1 d). In addition, the meniscus HE staining exhibited the same characteristics in PLB groups as the AMB groups (Fig. 2 a). After two weeks, the meniscus was structurally intact, the surface was poorly formed, it was pale yellow, the collagen fiber was worse and exhibited relaxation. The surface had poor flatness, the tissue was loose and showed irregular arrangement of chondrocyte cells (Fig. 2 b). After four weeks, the flatness of the meniscus was weak, and local slag formation was observed. The sequence of the collagen tissue had changed obviously, and a small amount of inflammatory cells had infiltrated into the meniscus (Fig. 2 c). After eight weeks, the smoothness of the meniscus was worse, the tissue was loose, the sequence of the collagen tissue was altered and the thickness was uneven. A large number of inflammatory cells had also infiltrated into the meniscus (Fig. $2 \mathrm{~d}$ ).

\section{Expressions of inflammatory factors in knee joint fluid of different groups}

Both IL-1 $\beta$ and IL-17 in the synovial fluid showed a demonstrable increase treads from the 2 nd to the 8th week in the ALB and PLB groups. Compared with the control group, there was a difference between ALB 
and PLB groups at three time phases $(P<0.05)$ as showed in Table 1 and Table 2.

\section{Levels of MMP-13 and MMP-13 mRNA in the meniscus of different groups}

Immunohistochemical staining for MMP-13 showed the same characteristics in the experimental groups. MMP-13 showed weak positive expression, both the cytoplasm and matrix were not expressed in the control groups. The surface, matrix and cytoplasm showed weak positive staining, the cells were rounded, full, and exhibited some cytoplasmic expressions for the 2 nd week. The expressions of the surface and interstitial inside the specimen showed strong positive staining, partial degradation of the matrix, relaxation of collagen fibrous tissue and change in cell morphology at the 4th week. Staining degree of meniscus decreased from 8 weeks, especially cytoplasm, matrix degradation, partial collagen fiber fracture, cell size and shape were different.

MMP-13 positive cells were counted in 100 images and compared in the three groups. The positive rate of MMP-13 expression in the AMB and PLB groups showed an increase treads from the 2nd to the 4th week. Compared with the control group, a significant difference was observed among 2, 4 and 8 weeks $(P<0.05)$. However, the positive rate of MMP-13 expression in the AMB and PLB groups showed a decrease after eight weeks (Table 1, Table 2).

The expressions of MMP-13 mRNA in the meniscus of the three groups were identified by RT-PCR. The findings demonstrated that the expression of MMP-13 mRNA increased from the 2nd week to peak at the 4 th week and the difference was significant $(P<0.05)$ compared to the control group on the AMB group and the PLB group. The level of MMP-13 mRNA decreased in the eighth week in both groups, although a significant difference was found $(P>0.05)$ compared to the control group (Fig. 3 ).

\section{Discussion}

The ACL is an important stable structure in the knee joint. Its principal role is tantamount to limit the tibial advance and to adjust the stability of the knee joint rotation function [11]. However, the ACL is also the most easily damaged structure in the knee joint [2]. Anatomically, the ACL can be subdivided into the $A M B$ and the PMB [12]. Each function bundle affects the tibial tuberosity advancement and rotation in different tension modes over the entire flexion and extension of the knee joint. If any of these band, it affects the stress distribution for all parts of the knee and can lead to damaging [13, 14]. As the anatomical structure and histochemistry of rabbit knee joints are similar to humans, rabbits were served as experimental models. Knee modeling was utilized to study the pathological process, histopathological features and cartilage biochemical metabolism of articular cartilage after partial ACL injury $[15,16]$.

In this study, we successfully established a partial ACL injury model in rabbit knee joint and systematically observed a serial of inflammatory effects on the meniscus at the second, fourth and eighth weeks. The complete healthy meniscus showed bright, white and smooth. When ACL injury on the discrete bundles occurred, the part of meniscus exhibited gradually unevenness and loose, whose surface become rough and yellow, afterwards, the deteriorated meniscus become relaxed. In a viewpoint of HE 
staining findings, the injuries of different functional bundles of the ACL were clearly identified as cause damage to the meniscus. More severe meniscus damages were associated with prolongation of the ACL injury. Immunohistochemistry results showed that MMP-13 was involved in the pathogenesis of meniscus degeneration. Due to the massive damage and death of chondrocytes according to the results of the pathological morphology of meniscus, inflammatory cells increased over time in this study, the amount of MMP-13 decreased accordingly. It is worth noting that the low expression of MMP13 did not necessarily represent the end of injury and chondrocyte apoptosis.

The meniscus lies in the articular space of the knee joint and also is a key structure of the knee joint. The meniscus acts transfer load and absorbs shock to maintain joint stability which results from vital functions of collagen which is composed of fibrous tissue [17]. The medial collateral ligament is contacted to the medial meniscus, and the fiber is connected with the diaphragm muscle [18]. The meniscus maintains stability and flexibility of the normal physiological function. Some studies have revealed that the knee joint can change the mechanical properties of the medial meniscus after injury of the knee at different angles [19]. When the meniscus angle is considerably higher than the anterior horn, the knee joint tomography suggests that the meniscus of the posterior horn is vulnerable to damage. This can lead to secondary knee medial meniscus damage which requires ACL reconstruction as soon as possible [20].

In the course of knee OA after ACL injury, inflammatory mediators play an important role at the beginning of the process and mechanical factors accelerate progression of the OA [21]. The pathogenesis of ACL injury leading to OA has not been fully elucidated. However, the rotational changes occurring after ACL injury may be a factor in the onset of knee $O A$ in healthy cartilage as a result of the shift of load transfer to the region that does not have frequently load bearing at the tibial-femoral contact of the articular surface during walking. Then, the shift in the load bearing area cause cartilage damage and increase fibrillation of collagen networks and matrix consolidation because of loss of proteoglycans at the surface layer. Eventually, as the cartilage begins to degradation, the disruption of the joint progresses more rapidly with increasing load. The degraded cartilage will produce upregulation of catabolic factors such as MMP and ILs. This finding was in an agreement with the publication of Andriacchi et al [22]. Studying the mechanism can help physicians choose appropriate treatments for patients including surgery, drugs and tissue engineering to reduce the incidence of OA [23].

In the present study, we measured the levels of IL-1 $\beta$ and IL-17 in synovial fluid concerning the role of inflammatory mediators in the process of chronic degeneration in the knee meniscus during eight weeks. Our findings indicated that the elevated levels of IL-1 $\beta$ and IL-17 suggested that inflammatory factors were involved in the degeneration of the meniscus. In previous studies, several studies have confirmed that IL-1 $\beta$ and IL-17 within synovial inflammation were associated with meniscus or cartilage degeneration as primary inflammatory mediators in humans or animals. Our study was in accord with these reporters $[24,25]$. High levels of IL-1 $\beta$ and IL-17 in knee traumatic areas demonstrated the level of meniscus or cartilage damage and the presence of a local inflammatory response that triggers early knee OA [26]. IL-17 is also a pro-inflammatory cytokine, which has been declared to increase synovitis and joint 
destruction following intra articular injection. IL-17 is mainly secreted by Th17 and can promote the proliferation and activation of T cells. Activated T cells develop a large number of cytokines such as IL$1 \beta$ to accelerate meniscus injury. IL-1 $\beta$ can inhibit the proliferation of chondrocytes by interfering with the metabolism of meniscus chondrocytes, and activate the MMPs signaling pathway. Studies indicated that a variety of inflammatory mediators play critical roles in the pathogenesis of knee trauma and OA progression. Expressions of various matrix MMPs are significantly increased, including MMP-1, MMP-2, MMP-3 and MMP-7 in addition to MMP-13 [27]. The increase in MMPs triggers a series of biological reactions that increase collagen degradation in the cartilage matrix and destroy meniscus integrity.

Current surgical treatment of the part ACL injury is different from bundle injury diagnosis, remains controversial $[28,29]$. The ACL part injury is a common ACL injury model, however, the treatment standards of patients with ACL injury are inconsistent [30,31]. An orthopedic expert reported that patients with ACL part injury who had been followed up for 9 to 15 years have developed a complete rupture, but only $32 \%$ of patients recovered to pre-injury levels. $48 \%$ of patients had a poor prognosis and $86 \%$ of patients had persistent symptoms [32]. The complete breakdown of the ACL single bundle injury may depend on the number of damaged fibers, the type of injury and the presence of secondary injury $[33,34]$. These factors may be particularly important after PLB injury and impact the stability of the knee joint $[35,36]$. According to the results of the study, the meniscus degradation began earlier in PLB groups compared to ALB groups.

There are some limitations to our study. First, the models were assessed at 2, 4 and 8 weeks, there remained a lack of long term follow-up observation till 12 weeks or more long time. Second, there was the absence of ACL reconstruction model to observe the effect on the meniscus. In addition, the study didn't include the histology quantification of any grading scale to evaluate the macroscopic appearance of the tissues. All these considerations will be validated in the future research.

\section{Conclusions}

We successfully established models with distinctive damage to functional bundle on ACL in the rabbit knee. Our findings illustrated that the elevated levels of IL-1 $\beta$ and IL-17 increased MMP13 expression in the posterior horn of the medial meniscus in the rabbit knee joint model with partial ACL injury, resulting in meniscus degradation and knee OA occurrence.

\section{Abbreviations}

ACL: anterior cruciate ligament; AMB: anteromedial bundle; PLB: posterolateral bundle; IL-1ß: interleukin13; IL-17: interleukin-17; OA: osteoarthritis; MMP: Matrix metalloproteinase; ELISA: enzyme-linked immunosorbant assay; CV: Coefficient of variation

\section{Declarations}


Acknowledgments

The study was partially presented in the International Conference on Applied Mechanics, Electronics and Mechantronics Engineering (AMEM 2017).

\section{Authors' contributions}

Dalin Wang conceived, designed the experiments and wrote a draft manuscript. Mingcheng Li analyzed, interpreted the results of the experiments and revised the manuscript. Zhe Wang and Songbao Xu performed the experiments. All authors read and approved the final manuscript.

\section{Funding}

This research was financially supported by the National Natural Science Foundation of China project (81402979), and received financial support from the Health commission research projects of Jilin Municipal, China (201830829) and Jilin Science and Technology Development Program (20200404036YY).

\section{Availability of data and materials}

All data generated or analyzed during this study are available in this published article.

\section{Ethics approval and consent to participate}

All experimental protocols conformed to the World Medical Association Declaration of Helsinki and were approved by the Animal Experiments Ethics Committee of the Associated Hospital of Beihua University (Ethical approval number: Protocol Number 2017-08-16).

\section{Consent for publication}

Not applicable

\section{Competing interests}

The authors declare that they have no competing interests. The authors alone are responsible for the content and writing of the paper.

\section{Author details}

${ }^{1}$ Dept. of Orthopaedic Surgery, Associated Hospital, Beihua University, Jilin, Jilin 132013, China

${ }^{2}$ Dept. of Clinical Diagnosis, School of Laboratory Medicine, Beihua University, Jilin, Jilin 132013, China

${ }^{3}$ Dept. of Orthopaedic Surgery, the First Hospital of National Petroleum Industry Co.Ltd, Jilin, China, 132015 


\section{References}

1. Rayan F, Nanjayan SK, Quah C, Ramoutar D, Konan S, Haddad FS. Review of evolution of tunnel position in anterior cruciate ligament reconstruction. World J Orthop. 2015; 6:252-262.

2. Zhang L, Guan TY, Qi J, Zhang SQ, Zhou X, Liu Y and Fu SJ. A model of anterior cruciate ligament injury in cynomolgus monkeys developed via arthroscopic surgery. EXP THER MED. 2018; 15: 22392246.

3. Wylie JD, Marchand LS, Burks RT. Etiologic factors that lead to failure after primary anterior cruciate ligament surgery. Clin Sports Med. 2017; 36(1):155-172.

4. Bryant AL, Kelly J, Hohmann E. Neuromuscular adaptations and correlates of knee functionality following ACL reconstruction. J Orthop Res. 2008; 26: 126-135.

5. Oiestad BE, Engebretsen L, Storheim K, Risberg MA. Knee osteoarthritis after anterior cruciate ligament injury: a systematic review. Am J Sports Med. 2009; 37:1434-1443.

6. Bigoni M, Turati M, Zatti G, et al. Intra-articular cytokine levels in adolescent patients after anterior cruciate ligament tear. Mediators Inflamm. 2018; 28; 2018:4210593.

7. Lohmander LS, Roos H, Dahlberg L, Hoerrner LA, Lark MW. Temporal patterns of stromelysin-1, tissue inhibitor, and proteoglycan fragments in human knee joint fluid after injury to the cruciate ligament or meniscus. J Orthop Res. 1994;12(1):21-8.

8. Wu X, Kondragunta V, Kornman KS, et al. IL-1 receptor antagonist gene as a predictive biomarker of progression of knee osteoarthritis in a population cohort. Osteoarthritis Cartilage. 2013; 21(7):930938

9. Chaudhari AM, Briant PL, Bevill SL, Koo S, Andriacchi TP. Anterior cruciate reconstruction combined with valgus upper tibial osteotomy: 12 years follow-up. Knee. 2004; 11(6):431-437.

10. Cicuttini FM, Wluka AE, Wang Y, Davis SR, Hankin J, Ebeling P. Compartment differences in knee cartilage volume in healthy adults. J Rheumatol. 2002; 29: 554-556.

11. Sun $L, W u B$, Tian M, Liu BC, Luo YZ. The significance of remnant preservation in anterior cruciate ligament reconstruction. Chin J Sports 2012; 31(7): 577-589 (Abstract in English).

12. Buchanan TS, Lloyd DG, Manal K, Besier TF. Estimation of muscle forces and joint moments using a forward-inverse dynamics model. Med Sci Sports Exerc. 2005; 37: 1911-1916.

13. SasaharaJ, TakaoM, MiyamotoW, OguroK, MatsushitaT. Partial harvesting technique in anterior cruciate ligament reconstruction with autologous semitendinosus tendon to prevent a postoperative decrease in deep knee flexion torque. Knee. 2014;21(5):936-43.

14. Anand BS, Feller JA, Richmond AK, Webster KE. Return-to-sport outcomes after revision anterior cruciate ligament reconstruction surgery. Am J Sports Med. 2016; 44(3):580-584.

15. Andernord D, Desai N, Björnsson H, Ylander M, Karlsson J, Samuelsson K. Patient predictors of early revision surgery after anterior cruciate ligament reconstruction: a cohort study of 16,930 patients with 2-year follow-up. Am J Sports Med. 2015; 43(1):121-127. 
16. Anderson AF, Snyder RB, Lipscomb AB. Anterior cruciate ligament reconstruction: a prospective randomized study of three surgical methods. Am J Sports Med. 2001; 29(3):272-279.

17. Claes S, Vereecke E, Maes M, Victor J, Verdonk P, Bellemans J. Anatomy of the anterolateral ligament of the knee. J Anat. 2013; 223(4):321-328.

18. Dodds AL, Halewood C, Gupte CM, Williams A, Amis AA. The anterolateral ligament: anatomy, length changes and association with the Segond fracture. Bone Joint J. 2014; 96-B(3):325-331.

19. Doherty DB, Lowe WR. Meniscal root tears: identification and repair. Am J Orthop (Belle Mead NJ). 2016; 45(3):183-187.

20. Fukubayashi T, Torzilli PA, Sherman MF, Warren RF. An in vitro biomechanical evaluation of anteriorposterior motion of the knee: tibial displacement, rotation, and torque. J Bone Joint Surg Am. 1982; 64(2):258-264.

21. Gossner J. The anterolateral ligament of the knee-visibility on magnetic resonance imaging. Rev Bras Ortop. 2014; 49(1):98-99.

22. Andriacchi TP, Mundermann A, Smith RL, Alexander EJ, Dyrby CO, Koo S. A framework for the in vivo pathomechanics of osteoarthritis at the knee. Ann Biomed Eng. 2004; 32: 447-457.

23. O’Malley MP, Milewski MD, Solomito MJ, Erwteman AS, Nissen CW. The association of tibial slope and anterior cruciate ligament rupture in skeletally immature patients. Arthroscopy. 2015; 31(1):7782.

24. Papageorgiou CD, Gil JE, Kanamori A, Fenwick JA, Woo SL-Y, Fu FH. The biomechanical interdependence between the anterior cruciate ligament replacement graft and the medial meniscus. Am J Sports Med. 2001; 29(2):226-231.

25. Savarese E, Bisicchia S, Romeo R, Amendola A. Role of high tibial osteotomy in chronic injuries of posterior cruciate ligament and posterolateral corner. J Orthop Traumatol. 2011; 12(1):1-17.

26. Shao Q, MacLeod TD, Manal K, Buchanan TS. Estimation of ligament loading and anterior tibial translation in healthy and ACL-deficient knees during gait and the influence of increasing tibial slope using EMG-driven approach. Ann Biomed Eng. 2011;39(1):110-121.

27. Shoemaker SC, Markolf KL. The role of the meniscus in the anterior-posterior stability of the loaded anterior cruciate-deficient knee: effects of partial versus total excision. J Bone Joint Surg Am. 1986; 68(1):71-79.

28. Stephen JM, Halewood C, Kittl C, Bollen SR, Williams A, Amis AA. Posteromedial meniscocapsular lesions increase tibiofemoral joint laxity with anterior cruciate ligament deficiency, and their repair reduces laxity. Am J Sports Med. 2016; 44(2):400-408.

29. Tavlo M, Eljaja S, Jensen JT, Siersma VD, Krogsgaard MR. The role of the anterolateral ligament in ACL insufficient and reconstructed knees on rotatory stability: a biomechanical study on human cadavers. Scand J Med Sci Sports. 2016; 26(8):960-966.

30. Thein R, Boorman-Padgett J, Stone K, Wickiewicz TL, Imhauser CW, Pearle AD. Biomechanical assessment of the anterolateral ligament of the knee: a secondary restraint in simulated tests of the pivot shift and of anterior stability. J Bone Joint Surg Am. 2016; 98(11):937-943. 
31. Todd MS, Lalliss S, Garcia E, DeBerardino TM, Cameron KL. The relationship between posterior tibial slope and anterior cruciate ligament injuries. Am J Sports Med. 2010; 38(1):63-67.

32. Bertrand SC, Lavoie F, Ogassawara R, Scussiato R. G, Kidder J. F Chambat P. Selective anteromedial bundle reconstruction in partial ACL tears: a series of 36 patients with mean 24 months follow-up. Knee Surg Sports Traumatol Arthrosc. 2010, 18:47-51.

33. Wassilew GI, Lehnigk U, Duda GN, et al. The expression of proinflammatory cytokines and matrix metalloproteinases in the synovial membranes of patients with osteoarthritis compared with traumatic knee disorders. Arthroscopy. 2010; 26(8): 1096-104.

34. Roberto B, Alberto F, Francesca V, Lisa Z, Francesco D C. Augmentation technique with semitendinosus and gracilistendons in chronic partial lesions of the ACL:Clinical and arthrometric analysis. Knee Surg Sports Traumatol Arthrosc. 2006, 14:1101-1107.

35. Anderson MJ, Diko S, Baehr LM, Baar K, Bodine SC, Christiansen BA. Contribution of mechanical unloading to trabecular bone loss following non-invasive knee injury in mice. J Orthop Res. 2016; 34:1680-1687.

\section{Tables}

Table 1 Comparison of MMP-13, IL-1 $\beta$ and IL-17 on AMB between the control and experimental groups ( $\mathrm{x} \pm \mathrm{s} \square \mathrm{n}=8$ )

\begin{tabular}{|c|c|c|c|c|c|c|c|}
\hline \multirow[t]{2}{*}{ Weeks } & \multicolumn{3}{|c|}{ Experimental group } & \multicolumn{3}{|c|}{ Control group } & \multirow[t]{2}{*}{$P$ value } \\
\hline & $\begin{array}{l}\text { MMP- } \\
13(\%)\end{array}$ & $\begin{array}{l}\text { IL-1 } \beta \\
(\mathrm{ng} / \mathrm{ml})\end{array}$ & $\begin{array}{l}\text { IL- } \\
17(\mathrm{ng} / \mathrm{ml})\end{array}$ & MMP-13(\%) & $\mathrm{IL}-1 \beta(\mathrm{ng} / \mathrm{ml})$ & $\mathrm{IL}-17(\mathrm{ng} / \mathrm{ml})$ & \\
\hline 2 & $1.56 \pm 0.21$ & $17.54 \pm 4.60$ & $20.31 \pm 8.45$ & $1.28 \pm 0.17$ & $1.52 \pm 1.60$ & $1.31 \pm 0.55$ & $\square 0.05^{*}$ \\
\hline 4 & $12.24 \pm 0.13$ & $21.44 \pm 6.70$ & $24.41 \pm 7.35$ & $1.47 \pm 0.21$ & $1.54 \pm 1.70$ & $1.33 \pm 0.45$ & $\square 0.05^{*}$ \\
\hline 8 & $10.53 \pm 0.25$ & $25.53 \pm 6.30$ & $38.31 \pm 6.53$ & $1.17 \pm 0.16$ & $1.53 \pm 1.50$ & $1.32 \pm 0.55$ & $\square 0.05^{*}$ \\
\hline
\end{tabular}

AMB: Anteromedial bundle

MMP-13: Matrix metallo proteinase-13

Data are presented as mean \pm standard deviation of three independent experiments, each in triplicates. ${ }^{* *} P<0.05$ compared with the sham control group determined by ANOVA and post hoc Bonferroni multiple comparisons test.

Table 2 Comparison of MMP-13, IL-1 $\beta$ and IL-17 on PLB between the control and experimental groups (` $\mathrm{x} \pm \mathrm{s} \square$ $\mathrm{n}=8)$ 


\begin{tabular}{|c|c|c|c|c|c|c|c|}
\hline \multirow[t]{2}{*}{ Weeks } & \multicolumn{3}{|c|}{ Experimental group } & \multicolumn{3}{|c|}{ Control group } & \multirow{2}{*}{$\begin{array}{r}P \\
\text { value }\end{array}$} \\
\hline & $\begin{array}{l}\text { MMP- } \\
13(\%)\end{array}$ & $\begin{array}{l}\text { IL-1 } \beta \\
\text { (ng/ml) }\end{array}$ & $\begin{array}{c}\mathrm{IL}- \\
17(\mathrm{ng} / \mathrm{ml})\end{array}$ & $\begin{array}{l}\text { MMP- } \\
13(\%)\end{array}$ & $\begin{array}{l}\text { IL-1 } \beta \\
\text { (ng/ml) }\end{array}$ & $\begin{array}{l}\text { IL- } \\
17(\mathrm{ng} / \mathrm{ml})\end{array}$ & \\
\hline 2 & $1.76 \pm 0.17$ & $17.54 \pm 4.60$ & $20.31 \pm 8.45$ & $1.15 \pm 0.20$ & $1.52 \pm 1.60$ & $1.31 \pm 0.55$ & $\square 0.05^{*}$ \\
\hline 4 & $12.46 \pm 0.23$ & $21.44 \pm 6.70$ & $24.41 \pm 7.35$ & $1.26 \pm 0.16$ & $1.54 \pm 1.70$ & $1.33 \pm 0.45$ & $\square 0.05^{* *}$ \\
\hline 8 & $10.79 \pm 0.21$ & $25.53 \pm 6.30$ & $38.31 \pm 6.53$ & $1.13 \pm 0.25$ & $1.53 \pm 1.50$ & $1.32 \pm 0.55$ & $\square 0.05^{* *}$ \\
\hline
\end{tabular}

PLB: Posterolateral bundle

MMP-13: Matrix metallo proteinase-13

Data are presented as mean \pm standard deviation of three independent experiments, each in triplicates.

** $P<0.05$ compared with the sham control group determined by ANOVA and post hoc Bonferroni multiple comparisons test.

Figures 


\section{a}

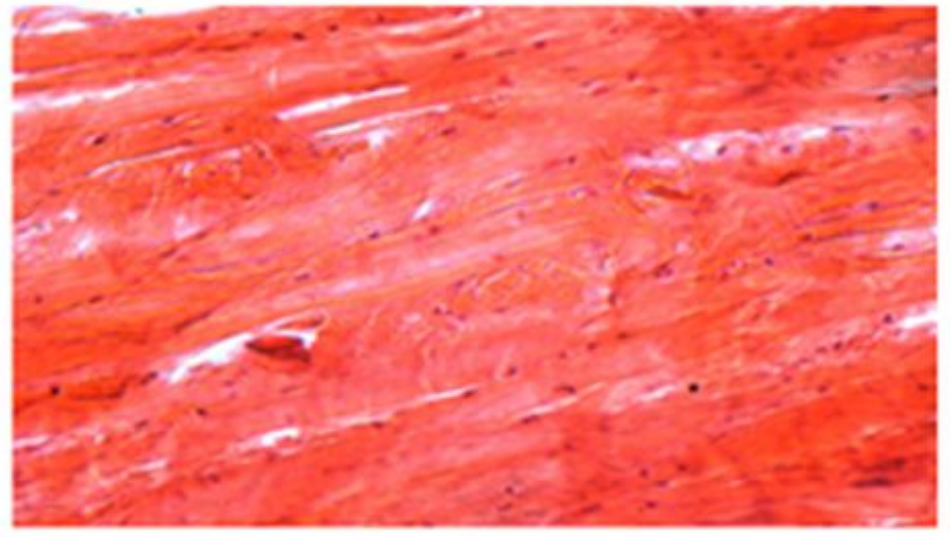

C

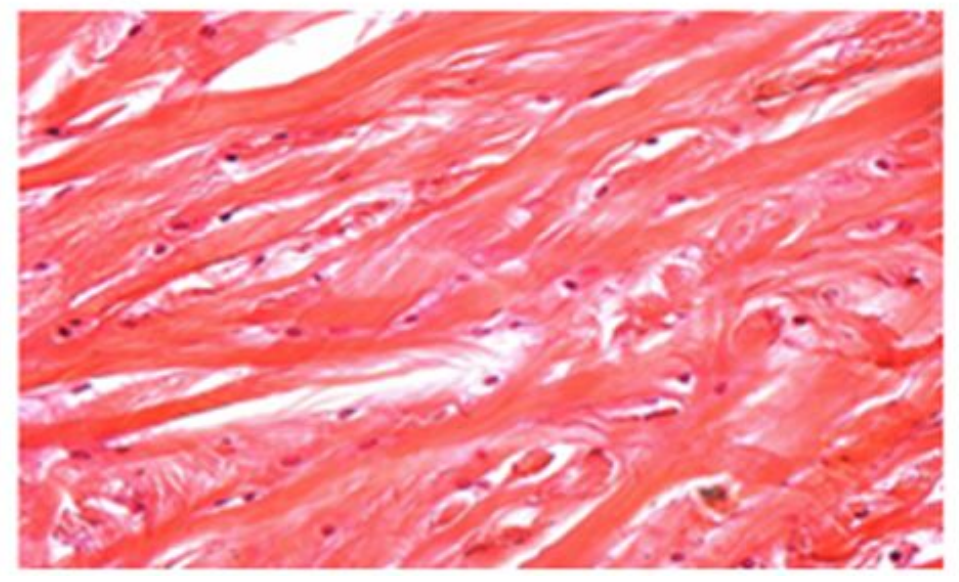

b

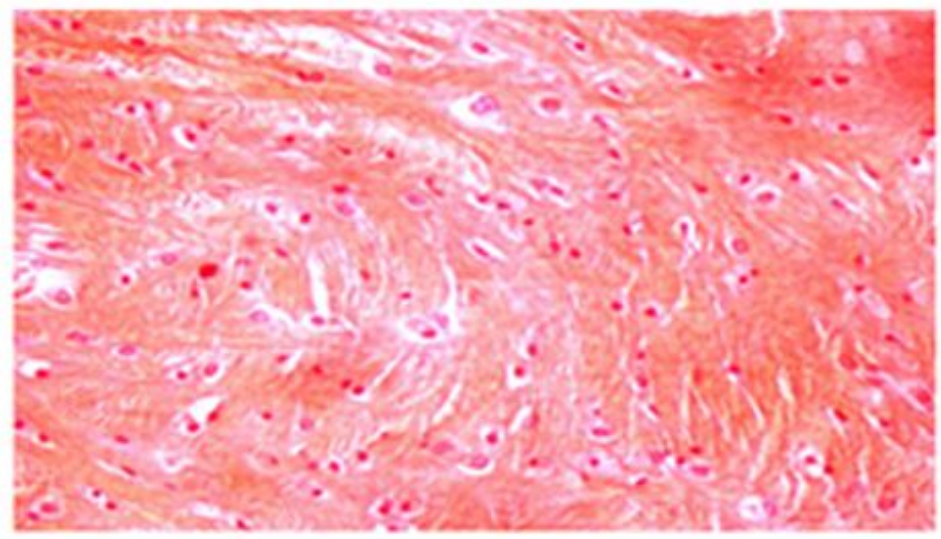

d

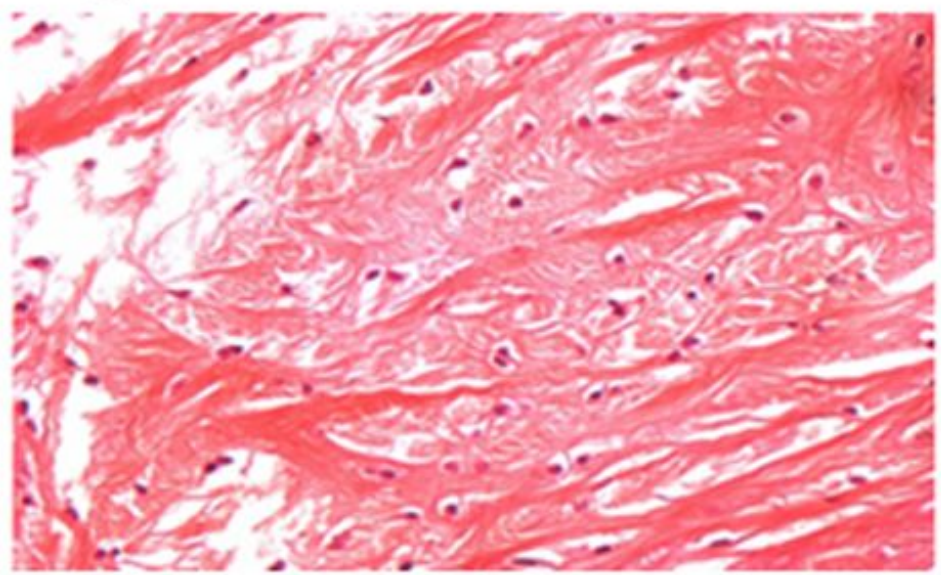

Figure 1

Morphologies of the knee meniscus and HE staining on the rabbits at the 2nd, 4th and 8th week after the operation in the AMB groups and control groups (HE staining $\times 400$ ). a. Structures of the knee meniscus and $\mathrm{HE}$ staining on the rabbits sacrificed after two weeks were imaged in the sham control groups. b. Structures of the knee meniscus and HE staining on the rabbits sacrificed after two weeks were imaged in the AMB groups. c. Structures of the knee meniscus and HE staining on the rabbits sacrificed after four weeks were imaged in the AMB groups. $d$. Structures of the knee meniscus and HE staining on the rabbits sacrificed after eight weeks were imaged in the AMB groups. AMB: Anteromedial bundle 


\section{a}

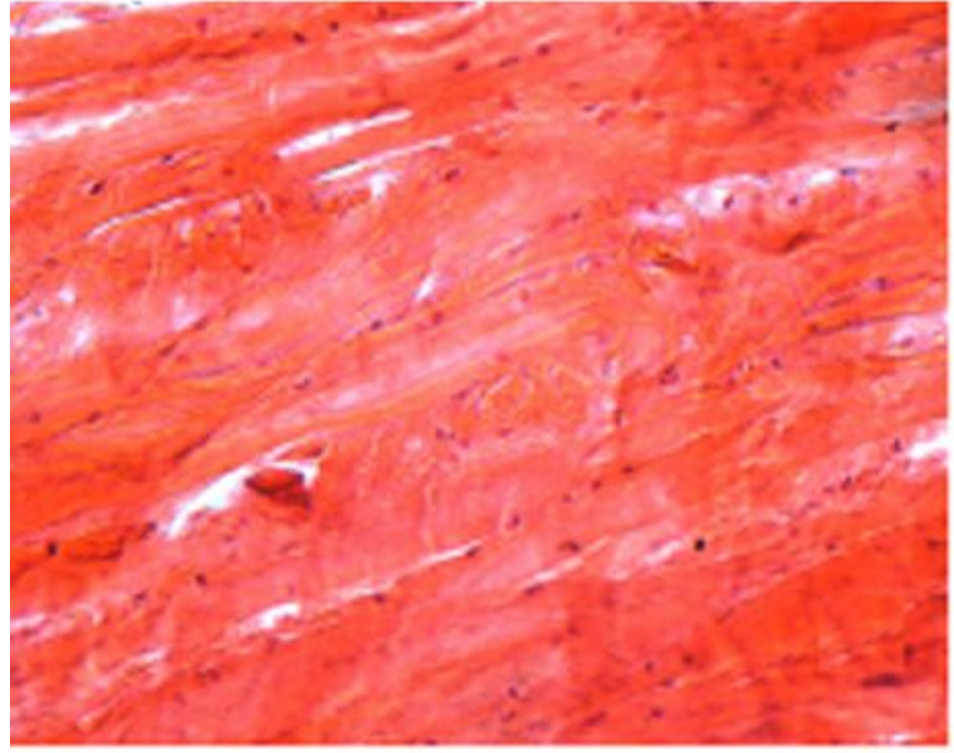

c

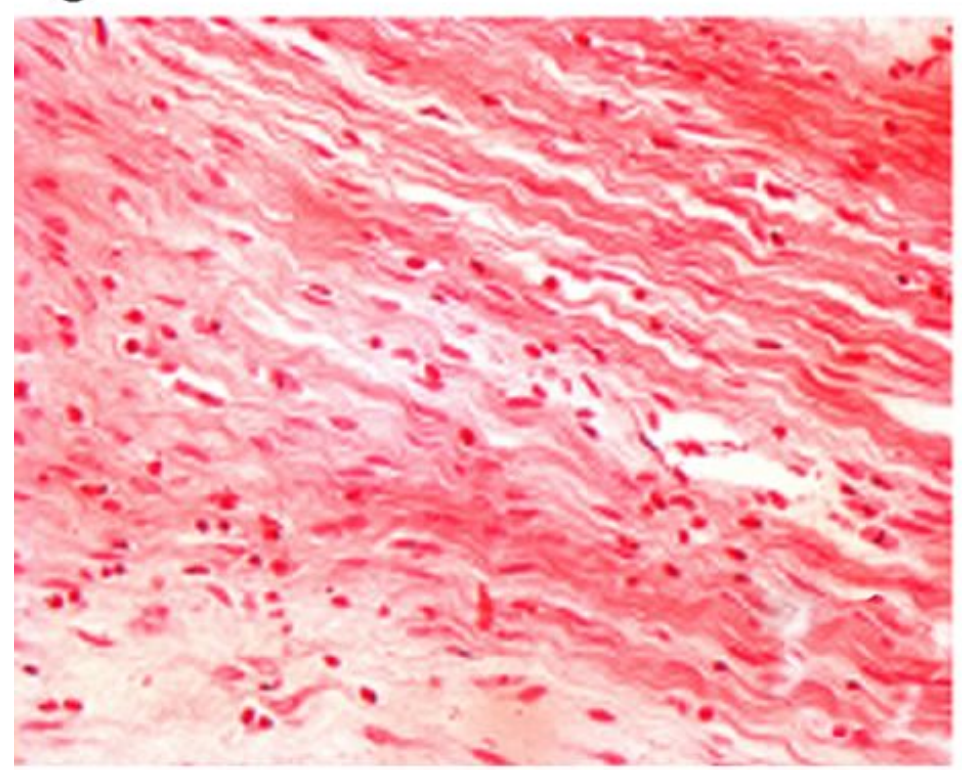

b

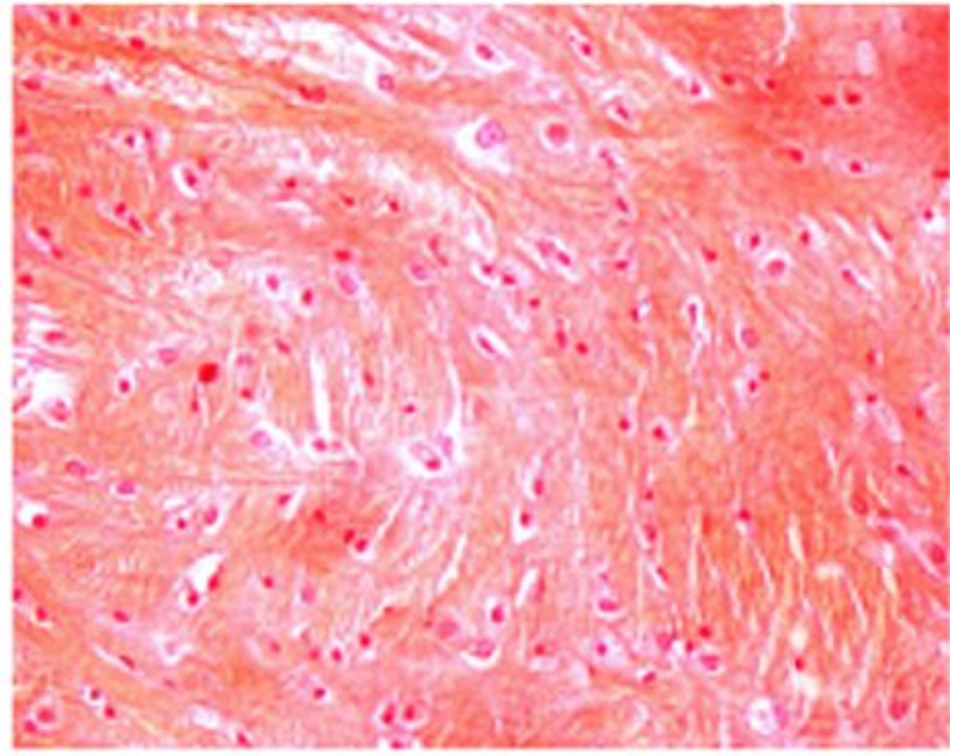

\section{d}

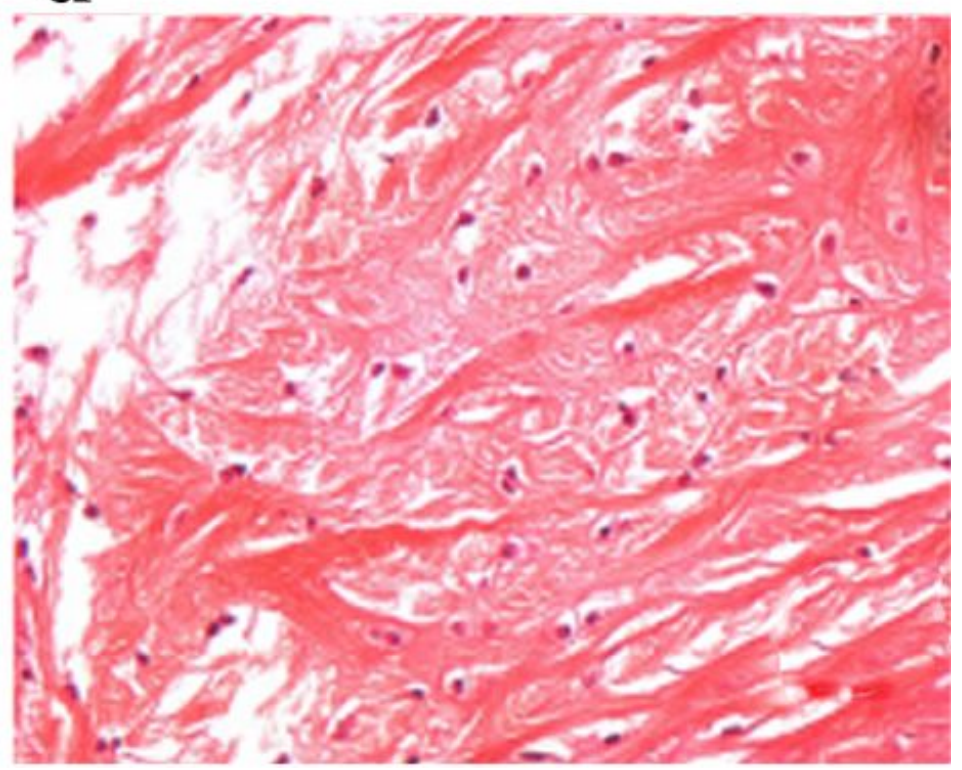

Figure 2

Morphologies of the knee meniscus and HE staining on the rabbits at the 2nd, 4th and 8th weeks after the operation in the PLB groups and sham control groups (HE staining, $\times 400$ ). a. Structures of the knee meniscus and HE staining on the rabbits sacrificed after two weeks were imaged in the sham control groups $b$. Structures of the knee meniscus and HE staining on the rabbits sacrificed after two weeks were imaged in the PLB groups. c. Structures of the knee meniscus and HE staining on the rabbits sacrificed after four weeks were imaged in the PLB groups. d. Structures of the knee meniscus and HE staining on the rabbits sacrificed after eight weeks were imaged in the PLB groups. PLB: Posterolateral bundle 

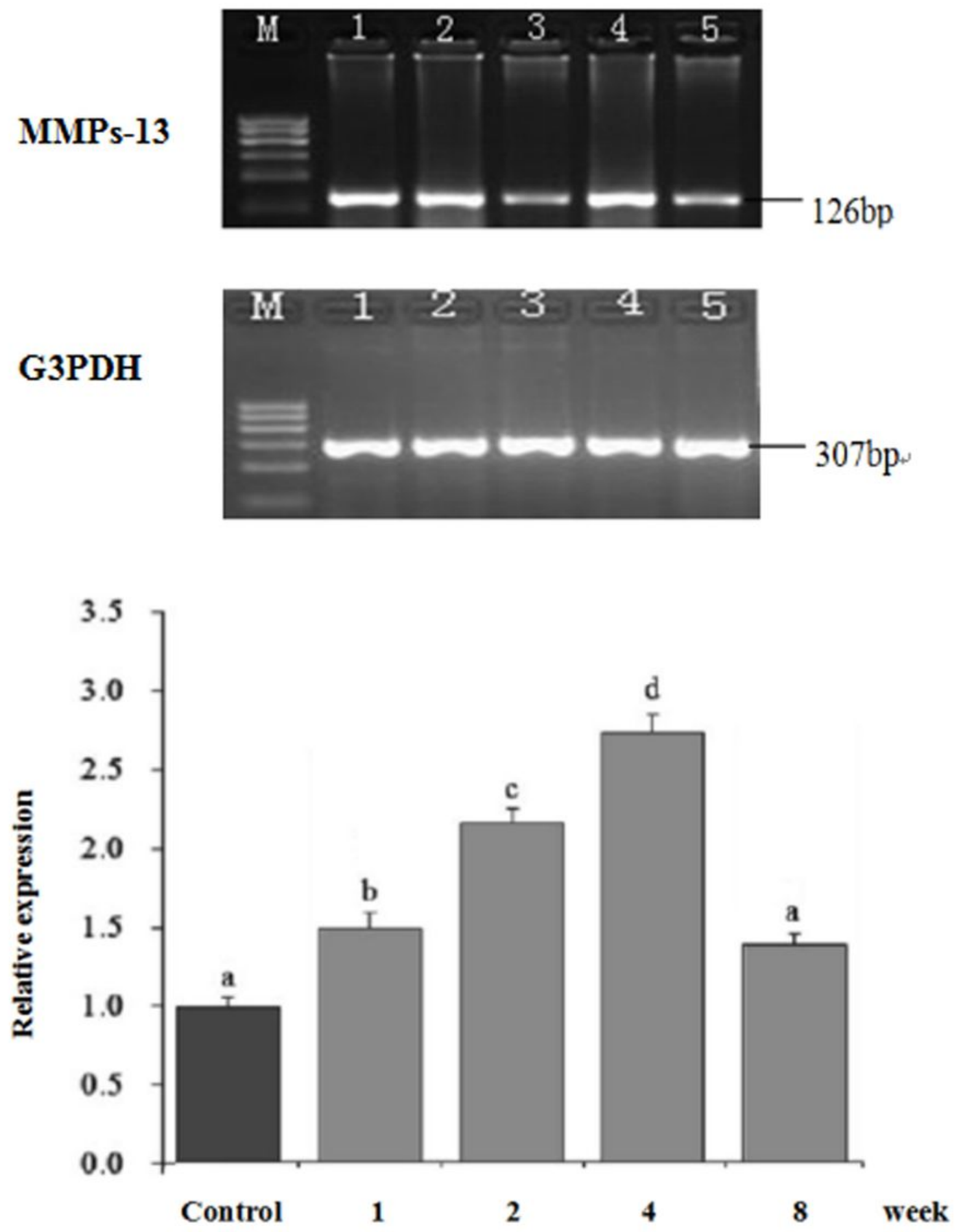

Figure 3

Levels of MMP-13 mRNA in the meniscus of different experimental groups. The expression of MMP-13 mRNA increased from the 2 nd week to the peak on the 4th week which was significantly different $(P<0.05)$ compared with the sham control group on the AMB group and the PLB groups. Whilst the level of MMP-13 mRNA decreased at the 8th week in both groups, the difference was not statistically 
significant $(P>0.05)$ compared with the sham control group. AMB: Anteromedial bundle; MMP-13: Matrix metalloproteinase-13 M: Marker; 